\title{
A Comparison of Archimedean Copula Models for approximating Bivariate Skew-Normal Distribution
}

\author{
A. Nanthakumar \\ Correspondence: A. Nanthakumar, State University of New York at Oswego, Oswego, NY 13126. E-mail: \\ ampala.nanthakumar@oswego.edu
}

Received: November 27, 2019 Accepted: December 30, 2019 Online Published: January 2, 2020

doi:10.5539/ijsp.v9n1p70

URL: https://doi.org/10.5539/ijsp.v9n1p70

\begin{abstract}
This paper compares the performance of some Archimedean Copulas in approximating the bivariate skew-normal distribution. Our study shows Frank Copula is a better Archimedean Copula for approximating the bivariate skew-normal distribution.
\end{abstract}

Keywords: skew-normal, bivariate, copula

\section{Introduction}

It has been noted in many applications that skewness is very prevalent in many univariate distributions which includes the family of normal distributions. A random variable $X$ is said to follow the skew-normal distribution if its density

$$
f(x, \lambda)=2 \phi(x) \Phi(\lambda x) \text { where } \lambda \text { is a constant. }
$$

where $\phi(x)$ and $\Phi(x)$ are the density function and the cumulative probability distribution function for the standard normal distribution respectively.

This skew-normal distribution and its applications have been studied extensively by Azzalini (1985, 1986), Henze (1986) and Quiroga (1992). The extension, bivariate skew-normal is also found to have a lot of applications. In this paper, the interest is in answering the question whether an Archimedean Copula could be used for approximating the bivariate skew-normal distribution while the marginal distributions are skew-normal distributions. If so, the interest is in finding the best Archimedean Copula that approximates the bivariate skew-normal distribution when the marginal distributions are skew-normal distributions. In fact, this paper aims to achieve this objective.

The concept of Copulas originated as a result of Sklar (1959) in probability theory. The Copulas allow for the construction of the joint distributions based on the marginal distributions. In the past, multivariate normal distributions dominated the study and analysis of multivariate data. However, recent literature reviews indicate a shift in this approach. For example, Krazanowski (1988) point out the need for alternatives to the normal distribution especially in actuarial science. This is an area where the copula models could fill the void. People are beginning to look at other variants of the normal distributions such as the folded normal, log-normal, skew-normal etc. Aziz (2001), Brown (2001), and Gupta et al(2004) investigated the properties and the applications of the skew-normal density functions. The multivariate skew-normal density functions arise in the context of economic and financial data. Charemza et al (2015) studied the use of the skew-normal distribution in the context of macro-economics. For the literature review please see Nelsen (2007).

In this paper, we investigate the possibility of fitting an Archimedean Copulas for the bivariate skew-normal density function. The aim is to identify the best fitting Archimedean Copula. Our research shows that "Frank Copula" is the best fitting Archimedean Copula for the bivariate skew-normal density function.

\section{Copulas}

Gaussian Copula:

$$
C\left(u_{1}, u_{2}\right)=\Phi_{2}\left(\Phi^{-1}\left(u_{1}\right), \Phi^{-1}\left(u_{2}\right)\right)
$$

where $\Phi_{2}(x, y)$ represents the bivariate standard normal cumulative distribution function and $\Phi^{-1}()$ represents the functional inverse for the standard normal cumulative distribution function. 


\section{Construction of Skew-Gaussian Copula:}

In this section, we investigate the properties of the skew-normal and the bivariate skew-normal distributions.

\section{Univariate Skew Normal:}

Result.

Let $U$ and $V$ be two independent standard normal variables and $\delta_{1}$ be a constant such that $-1<\delta_{1}<1$.

Then, $Z_{1}=\delta_{1} \cdot|U|+\sqrt{1-\delta_{1}^{2}} \cdot V$ follows a skew normal distribution.

Note that,

$$
\begin{gathered}
P\left(Z_{1} \leq z_{1}\right)=\int_{0}^{\infty} P\left(\sqrt{1-\delta_{1}^{2}} \cdot V \leq z_{1}-\delta_{1} \cdot u \backslash|U|=u\right) \cdot P(|U|=u) \cdot d u \\
=2 \cdot \int_{0}^{\infty} P\left(\sqrt{1-\delta_{1}^{2}} \cdot V \leq z_{1}-\delta_{1} \cdot u\right) \phi(u) \cdot d u=2 \cdot \int_{0}^{\infty} P\left(V \leq \frac{z_{1}}{\sqrt{1-\delta_{1}^{2}}}-\frac{\delta_{1} \cdot u}{\sqrt{1-\delta_{1}^{2}}}\right) \cdot \phi(u) \cdot d u \\
=2 \cdot \int_{0}^{\infty} P\left(V \leq \frac{z_{1}-a_{1} \cdot u}{b_{1}}\right) \cdot \phi(u) \cdot d u \\
F\left(z_{1}\right)=2 \cdot \int_{0}^{\infty} \Phi\left(\frac{\left(z_{1}-a_{1} \cdot u\right)}{b_{1}}\right) \cdot \phi(u) \cdot d u \\
\text { where } a_{1}=\delta_{1} \text { and } b_{1}=\sqrt{1-\delta_{1}^{2}} .
\end{gathered}
$$

Next, we will investigate the skew normal density function.

By differentiating with respect to $z_{1}$ we get,

$$
\begin{aligned}
& f\left(z_{1}\right)=\frac{2}{b_{1}} \cdot \int_{0}^{\infty} \phi\left(\frac{z_{1}-a_{1} \cdot u}{b_{1}}\right) \cdot \phi(u) \cdot d u \\
& =\frac{2}{b_{1}} \cdot \int_{0}^{\infty} \frac{1}{2 \cdot \pi} \cdot e^{-\frac{1}{2} \cdot\left(\frac{z_{1}-a_{1} \cdot u}{b_{1}}\right)^{2}-\frac{1}{2} \cdot u^{2}} \cdot d u \\
& =\frac{2}{b_{1} \cdot 2 \pi} \cdot \int_{0}^{\infty} e^{-\frac{1}{2} \cdot z_{1}^{2}} \cdot e^{-\frac{1}{2} \cdot\left(\frac{u-a_{1} \cdot z_{1}}{b_{1}}\right)^{2}} \cdot d u \\
& =2 \cdot \phi\left(z_{1}\right) \cdot\left(1-\Phi\left(\frac{-a_{1} \cdot z_{1}}{b_{1}}\right)\right)
\end{aligned}
$$

\section{Bivariate Skew-Normal}

Let $U, V$ be two independent standard normal variables and $U, W$ be two other independent standard normal variables along with constants $\delta_{1}, \delta_{2}$ such that $-1<\delta_{1}, \delta_{2}<1$.

$$
\begin{aligned}
& \text { Let } Z_{1}=\delta_{1} \cdot|U|+\sqrt{1-\delta_{1}^{2}} \cdot V \\
& Z_{2}=\delta_{2} \cdot|U|+\sqrt{1-\delta_{2}^{2}} \cdot W
\end{aligned}
$$

Then, $\left(Z_{1}, Z_{2}\right)$ follows a bivariate skew-normal distribution.

In order to derive the joint cumulative distribution $F\left(z_{1}, z_{2}\right)$, consider 


$$
\begin{gathered}
P\left(\delta_{1} \cdot|U|+\sqrt{1-\delta_{1}^{2}} \cdot V \leq z_{1}, \delta_{2} \cdot|U|+\sqrt{1-\delta_{2}^{2}} \cdot W \leq z_{2}\right) \\
=\int_{0}^{\infty} P\left(\sqrt{1-\delta_{1}^{2}} \cdot V \leq z_{1}-\delta_{1} \cdot u, \sqrt{1-\delta_{2}^{2}} \cdot W \leq z_{2}-\delta_{2} \cdot u \backslash|U|=u\right) \cdot P(|U|=u) \cdot d u \\
=2 \cdot \int_{0}^{\infty} P\left(\sqrt{1-\delta_{1}^{2}} \cdot V \leq z_{1}-\delta_{1} \cdot u, \sqrt{1-\delta_{2}^{2}} \cdot W \leq z_{2}-\delta_{2} \cdot u\right) \phi(u) \cdot d u
\end{gathered}
$$

This means, the joint cumulative skew normal distribution is

$$
F\left(z_{1}, z_{2}\right)=2 \cdot \int_{0}^{\infty} \Phi_{2}\left(\frac{z_{1}-a_{1} \cdot u}{b_{1}}, \frac{z_{2}-a_{2} \cdot u}{b_{2}}\right) \cdot \phi(u) \cdot d u
$$

where,

$$
a_{1}=\delta_{1}, b_{1}=\sqrt{1-\delta_{1}^{2}}
$$

and

$$
a_{2}=\delta_{2}, \quad b_{2}=\sqrt{1-\delta_{2}^{2}} .
$$

Note that the bivariate skew normal density is given by

$$
\begin{aligned}
& f\left(z_{1}, z_{2}\right)=\frac{\partial^{2} F\left(z_{1}, z_{2}\right)}{\partial z_{1} \cdot \partial z_{2}} \\
& =2 \cdot \int_{0}^{\infty} \frac{1}{b_{1} \cdot b_{2}} \cdot \phi_{2}\left(\frac{z_{1}-a_{1} \cdot u}{b_{1}}, \frac{z_{2}-a_{2} \cdot u}{b_{2}}\right) \cdot \phi(u) \cdot d u \\
& =\frac{2}{b_{1} \cdot b_{2}} \cdot \int_{0}^{\infty} \frac{1}{2 \cdot \pi \cdot \sqrt{1-\rho^{2}}} \cdot e^{-\frac{1}{2 \cdot\left(1-\rho^{2}\right)} \cdot\left\{\left(\frac{z_{1}-a \cdot u_{1}}{b_{1}}\right)^{2}+\left(\frac{z_{2}-a_{2} \cdot u}{b_{2}}\right)^{2}-2 \cdot \rho \cdot\left(\frac{z_{1}-a_{1} \cdot u}{b_{1}}\right) \cdot\left(\frac{z_{2}-a_{2} \cdot u}{b_{2}}\right)\right\}} \cdot \frac{1}{\sqrt{2 \cdot \pi}} \cdot e^{-\frac{1}{2} \cdot u^{2}} \cdot d u \\
& =2 \cdot \phi_{2}\left(z_{1}, z_{2}\right) \cdot \Phi\left(\frac{\left(a_{1} \cdot b_{2}^{2}-\rho \cdot a_{2} \cdot b_{1} \cdot b_{2}\right) \cdot z_{1}+\left(a_{2} \cdot b_{1}^{2}-\rho \cdot a_{1} \cdot b_{1} \cdot b_{2}\right) \cdot z_{2}}{\sqrt{\left(1-\rho^{2}\right) \cdot b_{1}^{2} \cdot b_{2}^{2}} \cdot \sqrt{a_{1}^{2} \cdot b_{2}^{2}+a_{2}^{2} \cdot b_{1}^{2}+b_{1}^{2} \cdot b_{2}^{2} \cdot\left(1-\rho^{2}\right)-2 \cdot \rho \cdot b_{1} \cdot b_{2} \cdot a_{1}^{2} \cdot a_{2}^{2}}}\right) \\
& e^{\frac{1}{2 \cdot\left(1-\rho^{2}\right)} \cdot\left\{\left[z_{1}^{2}-\left(\frac{z_{1}}{b_{1}}\right)^{2}\right]+\left[z_{2}^{2}-\left(\frac{z_{2}}{b_{2}}\right)^{2}\right]-2 \cdot \rho \cdot\left[z_{1} \cdot z_{2}-\left(\frac{z_{1} \cdot z_{2}}{b_{1} \cdot b_{2}}\right)\right]\right\}} \\
& \cdot e^{\frac{1}{2 \cdot\left(1-\rho^{2}\right) \cdot b_{1}^{2} \cdot b_{2}^{2}} \cdot\left\{\left(\frac{\left(a_{1} \cdot b_{2}^{2}-\rho \cdot a_{2} \cdot b_{1} \cdot b_{2}\right) \cdot z_{1}+\left(a_{2} \cdot b_{1}^{2}-\rho \cdot a_{1} \cdot b_{1} \cdot b_{2}\right) \cdot z_{2}}{\left(a_{1}^{2} \cdot b_{2}^{2}+a_{2}^{2} \cdot b_{1}^{2}+b_{1}^{2} \cdot b_{2}^{2} \cdot\left(1-\rho^{2}\right)-2 \cdot \rho \cdot a_{1}^{2} \cdot a_{2}^{2} \cdot b_{1} \cdot b_{2}\right)}\right\}\right.} \\
& =2 . \Phi\left(\frac{\left(a_{1} \cdot b_{2}^{2}-\rho \cdot a_{2} \cdot b_{1} \cdot b_{2}\right) \cdot z_{1}+\left(a_{2} \cdot b_{1}^{2}-\rho \cdot a_{1} \cdot b_{1} \cdot b_{2}\right) \cdot z_{2}}{\sqrt{\left(1-\rho^{2}\right) \cdot b_{1}^{2} \cdot b_{2}^{2}} \cdot \sqrt{a_{1}^{2} \cdot b_{2}^{2}+a_{2}^{2} \cdot b_{1}^{2}+b_{1}^{2} \cdot b_{2}^{2} \cdot\left(1-\rho^{2}\right)-2 \cdot \rho \cdot b_{1} \cdot b_{2} \cdot a_{1}^{2} \cdot a_{2}^{2}}}\right) \\
& \cdot e^{\frac{1}{2 \cdot\left(1-\rho^{2}\right) \cdot b_{1}^{2} \cdot b_{2}^{2}} \cdot\left\{\frac{\left(a_{1} \cdot b_{2}^{2}-\rho \cdot a_{2} \cdot b_{1} \cdot b_{2}\right) \cdot z_{1}+\left(a_{2} \cdot b_{1}^{2}-\rho \cdot a_{1} \cdot b_{1} \cdot b_{2}\right) \cdot z_{2}}{\left(a_{1}^{2} \cdot b_{2}^{2}+a_{2}^{2} \cdot b_{1}^{2}+b_{1}^{2} \cdot b_{2}^{2} \cdot\left(1-\rho^{2}\right)-2 \cdot \rho \cdot a_{1}^{2} \cdot a_{2}^{2} \cdot b_{1} \cdot b_{2}\right)}\right\}} \\
& e^{-\frac{1}{2 \cdot\left(1-\rho^{2}\right)} \cdot\left\{\left(\frac{z_{1}}{b_{1}}\right)^{2}+\left(\frac{z_{2}}{b_{2}}\right)^{2}-2 \cdot \rho \cdot\left(\frac{z_{1}}{b_{1}}\right) \cdot\left(\frac{z_{2}}{b_{2}}\right)\right\}}
\end{aligned}
$$

Let us investigate the mean, variance, skewness, kurtosis, and covariance of $Z_{1}$ and $Z_{2}$. 
Note that $Z_{1}=\delta_{1} \cdot|U|+\sqrt{1-\delta_{1}^{2}} \cdot V$

$E\left(Z_{1}\right)=\delta_{1} \cdot E(|U|)+\sqrt{1-\delta_{1}^{2}} \cdot E(V)$

Note that $E(|U|)=\int_{-\infty}^{0}-u \cdot f(u) d u+\int_{0}^{\infty} u \cdot f(u) d u$

$$
\begin{gathered}
=\int_{\infty}^{0} u \cdot f(-u) d(-u)+\int_{0}^{\infty} u \cdot f(u) d u \\
=\int_{0}^{\infty} u \cdot f(u) d u+\int_{0}^{\infty} u \cdot f(u) d u \\
=2 \cdot \int_{0}^{\infty} u \cdot f(u) d u \\
=\frac{2}{\sqrt{2 \pi}} \cdot \int_{0}^{\infty} e^{-\frac{u^{2}}{2}} \cdot d \frac{u^{2}}{2} \\
=\sqrt{\frac{2}{\pi}}
\end{gathered}
$$

This implies, $E\left(Z_{1}\right)=\delta_{1} \cdot \sqrt{\frac{2}{\pi}}$

Similarly,

$$
E\left(Z_{2}\right)=\delta_{2} \cdot \sqrt{\frac{2}{\pi}}
$$

Also,

$$
\begin{gathered}
E\left(Z_{1}^{2}\right)=E\left\{\delta_{1}^{2} \cdot U^{2}+\left(1-\delta_{1}^{2}\right) \cdot V^{2}+2 \cdot \delta_{1} \cdot \sqrt{1-\delta_{1}^{2}} \cdot|U| \cdot V\right\} \\
=\delta_{1}^{2} \cdot E\left(U^{2}\right)+\left(1-\delta_{1}^{2}\right) \cdot E\left(V^{2}\right)+2 \cdot \delta_{1} \cdot \sqrt{1-\delta_{1}^{2}} \cdot E(|U| \cdot V) \\
=\delta_{1}^{2}+1-\delta_{1}^{2} \\
=1 \\
\operatorname{Var}\left(Z_{1}\right)=E\left(Z_{1}^{2}\right)-\left(E\left(Z_{1}\right)\right)^{2} \\
=1-\frac{2}{\pi} \cdot \delta_{1}^{2}
\end{gathered}
$$

Similarly,

$$
\operatorname{Var}\left(Z_{2}\right)=1-\frac{2}{\pi} \cdot \delta_{2}^{2}
$$

Note that, $\operatorname{Cov}\left(Z_{1}, Z_{2}\right)=E\left(Z_{1} \cdot Z_{2}\right)-E\left(Z_{1}\right) \cdot E\left(Z_{2}\right)$

and

$$
\begin{gathered}
Z_{1} \cdot Z_{2}=\left\{\delta_{1} \cdot|U|+\sqrt{1-\delta_{1}^{2}} \cdot V\right\}\left\{\delta_{2} \cdot|U|+\sqrt{1-\delta_{2}^{2}} \cdot W\right\} \\
=\delta_{1} \cdot \delta_{2} \cdot U^{2}+\sqrt{1-\delta_{1}^{2}} \cdot \delta_{2} \cdot|U| \cdot V+\delta_{1} \cdot \sqrt{1-\delta_{2}^{2}} \cdot|U| \cdot W+\sqrt{1-\delta_{1}^{2}} \cdot \sqrt{1-\delta_{2}^{2}} \cdot V \cdot W
\end{gathered}
$$

This means that 


$$
E\left(Z_{1} \cdot Z_{2}\right)=\delta_{1} \cdot \delta_{2}+\sqrt{1-\delta_{1}^{2}} \cdot \sqrt{1-\delta_{2}^{2}} \cdot \rho_{v, w}
$$

So, $\operatorname{Cov}\left(Z_{1}, Z_{2}\right)=\delta_{1} \cdot \delta_{2}+\sqrt{1-\delta_{1}^{2}} \cdot \sqrt{1-\delta_{2}^{2}} \cdot \rho_{v, w}-\frac{2}{\pi} \cdot \delta_{1} \cdot \delta_{2}$

$$
=\delta_{1} \cdot \delta_{2} \cdot\left(1-\frac{2}{\pi}\right)+\sqrt{1-\delta_{1}^{2}} \cdot \sqrt{1-\delta_{2}^{2}} \cdot \rho_{v, w}
$$

It is obvious that the correlation coefficient for the relationship between $Z_{1}$ and $Z_{2}$ is

$$
\rho_{Z_{1}, Z_{2}}=\frac{\left(1-\frac{2}{\pi}\right) \cdot \delta_{1} \cdot \delta_{2}+\sqrt{1-\delta_{1}^{2}} \cdot \sqrt{1-\delta_{2}^{2}} \cdot \rho_{v, w}}{\sqrt{1-\frac{2}{\pi} \cdot \delta_{1}^{2}} \cdot \sqrt{1-\frac{2}{\pi} \cdot \delta_{2}^{2}}}
$$

Note:

For the skew-normal distribution, the moment generating function is

$$
\mathrm{M}(t)=2 \cdot(1-\Phi(-t . \delta)) \cdot e^{\frac{t^{2}}{2}}
$$

Furthermore,

Also,

$$
\begin{gathered}
E\left(Z^{3}\right)=\left(3-\delta^{2}\right) \cdot \delta \cdot \sqrt{\frac{2}{\pi}} \\
E\left(Z^{4}\right)=3
\end{gathered}
$$

$$
\begin{gathered}
E\left((Z-\mu)^{3}\right)=E\left(Z^{3}\right)-3 \cdot \mu \cdot E\left(Z^{2}\right)+3 \cdot \mu^{2} \cdot E(Z)-\mu^{3} \\
=\left(3-\delta^{2}\right) \cdot \delta \cdot \sqrt{\frac{2}{\pi}}-3 \cdot \delta \cdot \sqrt{\frac{2}{\pi}}+2 \cdot \delta^{3} \cdot\left(\frac{2}{\pi}\right)^{\frac{3}{2}} \\
=\sqrt{\frac{2}{\pi}} \cdot\left(\frac{4}{\pi}-1\right) \cdot \delta^{3} \\
E\left((Z-\mu)^{4}\right)=E\left(Z^{4}\right)-4 \cdot \mu \cdot E\left(Z^{3}\right)+6 \cdot \mu^{2} \cdot E\left(Z^{2}\right)-4 \cdot \mu \cdot E(Z)+\mu^{4} \\
=3-4 \cdot\left(\frac{2}{\pi}\right) \cdot \delta^{2} \cdot\left(3-\delta^{2}\right)+6 \cdot\left(\frac{2}{\pi}\right) \cdot \delta^{2}-4 \cdot\left(\frac{2}{\pi}\right) \cdot \delta^{2}+\left(\frac{2}{\pi}\right)^{2} \cdot \delta^{4} \\
=3-\left(\frac{20}{\pi}\right) \cdot \delta^{2}+\left(\frac{8}{\pi}+\frac{4}{\pi^{2}}\right) \cdot \delta^{4}
\end{gathered}
$$

Skewness $\beta=\frac{E(Z-\mu)^{3}}{\left(E(Z-\mu)^{2}\right)^{\frac{3}{2}}}$

Kurtosis, $\kappa=\frac{E\left((Z-\mu)^{4}\right)}{\left(E(Z-\mu)^{2}\right)^{2}}$

$$
=\frac{\sqrt{\frac{2}{\pi}} \cdot\left(\frac{4}{\pi}-1\right) \cdot \delta^{3}}{\left(1-\frac{2}{\pi} \cdot \delta^{2}\right)^{\frac{3}{2}}}
$$




$$
=\frac{\left(3-\left(\frac{20}{\pi}\right) \cdot \delta^{2}+\left(\frac{8}{\pi}+\frac{4}{\pi^{2}}\right) \cdot \delta^{4}\right)}{\left(1-\frac{2}{\pi} \cdot \delta^{2}\right)^{2}}
$$

Next, we present a theorem (see Genest and MacKay (1986) to check whether the Archimedean Copulas are adequate to fit a bivariate skew-normal distribution.

Theorem (Genest \& MacKay):

Let $Z_{1}$ and $Z_{2}$ be continuous random variables with their joint distribution function represented by the Copula $C\left(z_{1}, z_{2}\right)$.

Then, $P\left(C\left(z_{1}, z_{2}\right) \leq z\right)=z-\frac{\phi(z)}{\phi^{\prime}(z)}$ where $\phi(z)$ is the Copula generator function.

Next, we will investigate which Archimedean Copula is the better for modeling the bivariate skew-normal distribution.

\section{Clayton Copula:}

$$
\begin{aligned}
& C\left(z_{1}, z_{2}\right)=C\left(u_{1}, u_{2}\right) \\
& =\phi^{-1}\left(\phi\left(u_{1}\right)+\phi\left(u_{2}\right)\right)
\end{aligned}
$$

where $\phi(u)$ is the generator function.

Note that $u_{1}$ and $u_{2}$ represent the marginal probability distribution functions of $Z_{1}$ and $Z_{2}$ respectively.

For the Clayton Copula, $\phi(u)=u^{-\theta}-1$

This means, $C\left(z_{1}, z_{2}\right)=\left(u_{1}^{-\theta}+u_{2}^{-\theta}-1\right)^{\frac{-1}{\theta}}$

Note that for the Clayton Copula, the correlation coefficient

$$
\rho_{Z_{1}, Z_{2}} \approx \frac{\theta}{\theta+2}
$$

Due to Genest and MacKay Theorem,

$$
\begin{aligned}
& P\left(C\left(z_{1}, z_{2}\right) \leq z\right)=z-\frac{\phi(z)}{\phi^{\prime}(z)} \\
& \quad=z \cdot\left(1+\frac{1}{\theta}-\frac{z^{\theta}}{\theta}\right)
\end{aligned}
$$

\section{Gumbel Copula:}

$$
\begin{aligned}
& C\left(z_{1}, z_{2}\right)=C\left(u_{1}, u_{2}\right) \\
& =\phi^{-1}\left(\phi\left(u_{1}\right)+\phi\left(u_{2}\right)\right)
\end{aligned}
$$

where $\phi(u)$ is the Copula generator function.

As noted earlier, $u_{1}$ and $u_{2}$ represent the marginal probability distribution functions of $Z_{1}$ and $Z_{2}$ respectively.

For the Gumbel Copula, $\phi(u)=(-\ln (u))^{-\theta}$

This means, $C\left(u_{1}, u_{2}\right)=e^{-\left[\left(-\ln u_{1}\right)^{\theta}+\left(-\ln u_{2}\right)^{\theta}\right]^{\frac{1}{\theta}}}$

Note that for the Gumbel Copula, the correlation coefficient

$$
\rho_{Z_{1}},_{Z_{2}} \approx 1-\theta^{-1}
$$




$$
\text { Again, } \begin{aligned}
P( & \left.C\left(z_{1}, z_{2}\right) \leq z\right)=z-\frac{\phi(z)}{\phi^{\prime}(z)} \\
& =z-\frac{z}{\theta} \cdot(-\ln (z)) \\
& =z \cdot\left(1+\frac{1}{\theta} \cdot \ln (z)\right)
\end{aligned}
$$

\section{Frank Copula:}

$$
C\left(z_{1}, z_{2}\right)=C\left(u_{1}, u_{2}\right)
$$

$=\phi^{-1}\left(\phi\left(u_{1}\right)+\phi\left(u_{2}\right)\right)$ where $\phi(u)$ is the generator function.

As noted earlier, $u_{1}$ and $u_{2}$ represent the marginal probability distribution functions of $Z_{1}$ and $Z_{2}$ respectively.

For the Frank Copula, $\phi(u)=\ln \left\{\frac{\left(e^{\theta . t}-1\right)}{\left(e^{\theta}-1\right)}\right\}$

This means, $C\left(u_{1}, u_{2}\right)=\frac{1}{\theta} \cdot \ln \left(1+\frac{\left(e^{\theta \cdot u_{1}}-1\right) \cdot\left(e^{\theta \cdot u_{2}}-1\right)}{\left(e^{\theta}-1\right)}\right)$

Note that for the Frank Copula, the correlation coefficient $\rho_{Z_{1}, Z_{2}}=1-\frac{12}{\theta} \cdot\left[D_{2}(-\theta)-D_{1}(-\theta)\right]$

where $D_{k}(x)=\frac{k}{x^{k}} \cdot \int_{0}^{x} \frac{t^{k}}{\left(e^{t}-1\right)} d t$ for $k=1,2$ and it is called "Debye" function.

Note that $D_{k}(-x)=D_{k}(x)+\frac{k \cdot x}{k+1}$

So, $D_{1}(-\theta)=D_{1}(\theta)+\frac{\theta}{2}$ and $D_{2}(-\theta)=D_{2}(\theta)+\frac{2 \theta}{3}$

This means, $D_{2}(-\theta)-D_{1}(-\theta)=D_{2}(\theta)-D_{1}(\theta)+\frac{\theta}{6}$

This in turn yields

$$
\begin{gathered}
\rho_{Z_{1}, Z_{2}}=1-\frac{12}{\theta} \cdot\left[D_{2}(-\theta)-D_{1}(-\theta)\right] \\
=1-\frac{12}{\theta} \cdot\left[D_{2}(\theta)-D_{1}(\theta)+\frac{\theta}{6}\right] \\
=1-\frac{12}{\theta} \cdot\left[D_{2}(\theta)-D_{1}(\theta)\right]-2 \\
=\frac{-12}{\theta} \cdot\left[D_{2}(\theta)-D_{1}(\theta)\right]-1
\end{gathered}
$$

This implies

$$
1+\rho_{Z_{1}}, z_{2}=\frac{-12}{\theta} \cdot\left[D_{2}(\theta)-D_{1}(\theta)\right]
$$

Note: We will use the above equation to estimate the dependence parameter $\theta$ for the Frank Copula.

Again note that, $P\left(C\left(z_{1}, z_{2}\right) \leq z\right)=z-\frac{\phi(z)}{\phi^{\prime}(z)}$ 


$$
=z-\frac{\left(e^{\theta \cdot z}-1\right)}{\theta \cdot e^{\theta \cdot z}} \cdot \ln \left\{\frac{\left(e^{\theta \cdot z}-1\right)}{\left(e^{\theta}-1\right)}\right\}
$$

\section{Numerical Resultsss}

Here in this section, we present the results based on samples of size $=1000$ which were also run 1000 times. This simulation study was done to compare the performance of Clayton, Gumbel, and Frank Copulas. The Copulas were compared on the basis of the distribution of the Copulas,

$$
P\left(C\left(z_{1}, z_{2}\right) \leq z\right)=z-\frac{\phi(z)}{\phi^{\prime}(z)}
$$

Note that the simulation uses the following parameters and $\phi($.$) is the generator function.$

$$
\begin{gathered}
\delta_{1}=\frac{\lambda_{1}}{\sqrt{1+\lambda_{1}^{2}}}, \quad \sqrt{1-\delta_{1}^{2}}=\frac{1}{\sqrt{1+\lambda_{1}^{2}}} \\
\delta_{2}=\frac{\lambda_{2}}{\sqrt{1+\lambda_{2}^{2}}}, \quad \sqrt{1-\delta_{2}^{2}}=\frac{1}{\sqrt{1+\lambda_{2}^{2}}} \\
\rho_{Z_{1}, Z_{2}}=\frac{\left(1-\frac{2}{\pi}\right) \cdot \delta_{1} \cdot \delta_{2}+\sqrt{1-\delta_{1}^{2}} \cdot \sqrt{1-\delta_{2}^{2}} \cdot \rho_{v, w}}{\sqrt{1-\frac{2}{\pi} \cdot \delta_{1}^{2}} \cdot \sqrt{1-\frac{2}{\pi} \cdot \delta_{2}^{2}}}
\end{gathered}
$$

Note 1: ssThe following simulations involve computing the left hand side of equation (30) empirically while computing the right hand side of equation (30) by using different copula models such as Clayton, Gumbel, and Frank. Note that the dependence parameter $(\theta)$ estimate is different for each copula that is investigated. The purpose is to find out which Archimedean Copula performs better in approximating a bivariate skew-normal distribution.

Note 2:

The following simulation tables indicate the dependence parameter $(\theta)$ estimate within horizontal brackets for the different copulas.

Note 3:

The graphs corresponding to different copulas have the following colors.

$$
\begin{aligned}
& \text { Clayton } \sim \text { Blue } \\
& \text { Gumbel } \sim \text { Red } \\
& \text { Frank } \sim \text { Green } \\
& \text { Empirical } \sim \text { Purple }
\end{aligned}
$$

\subsection{Simulation Tables and Graphs}

Simulation \#1

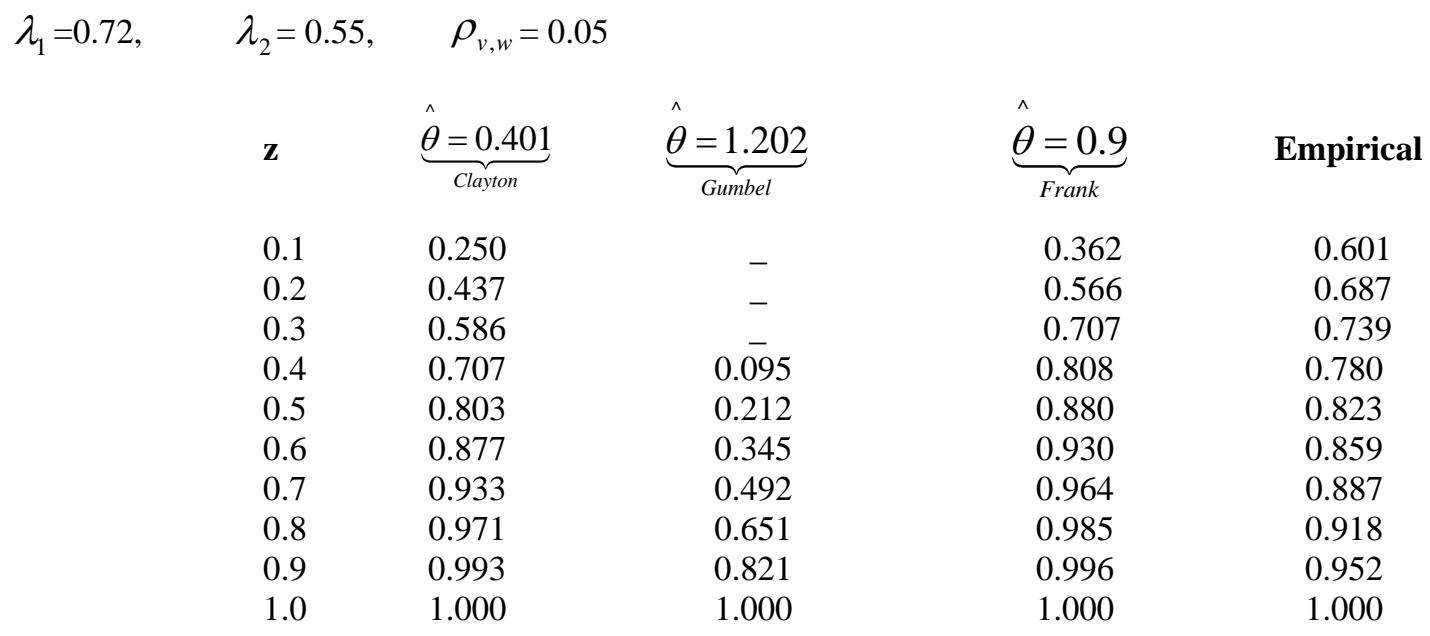




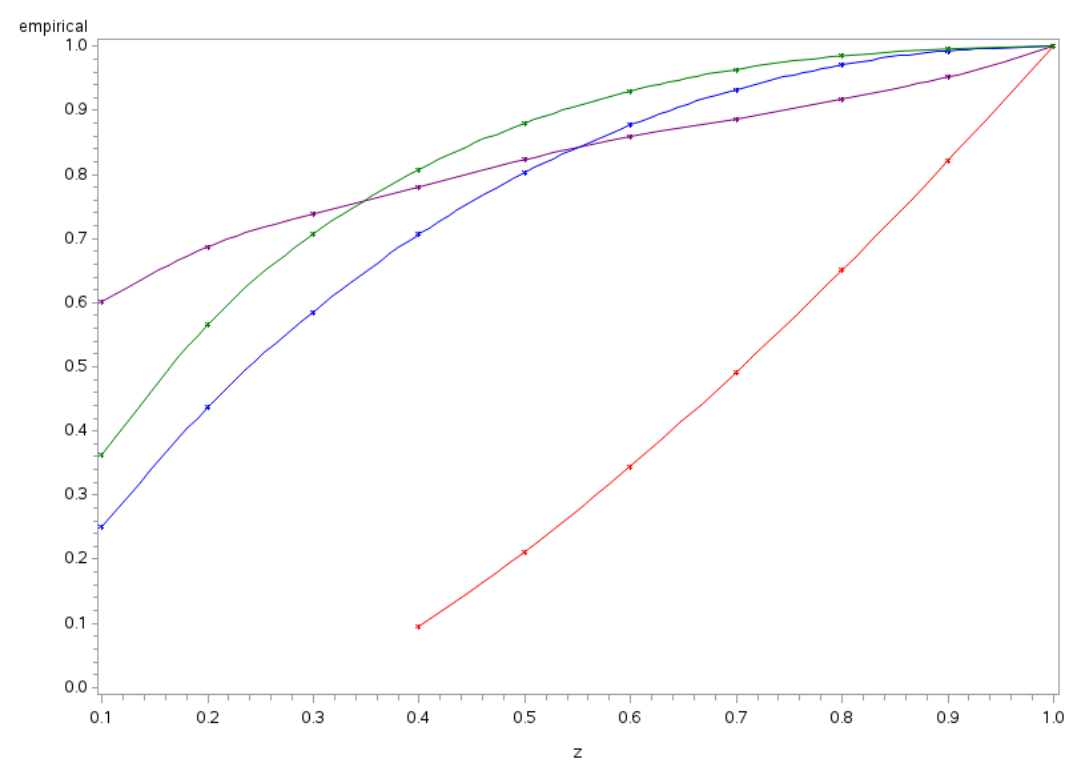

Simulation \# 2

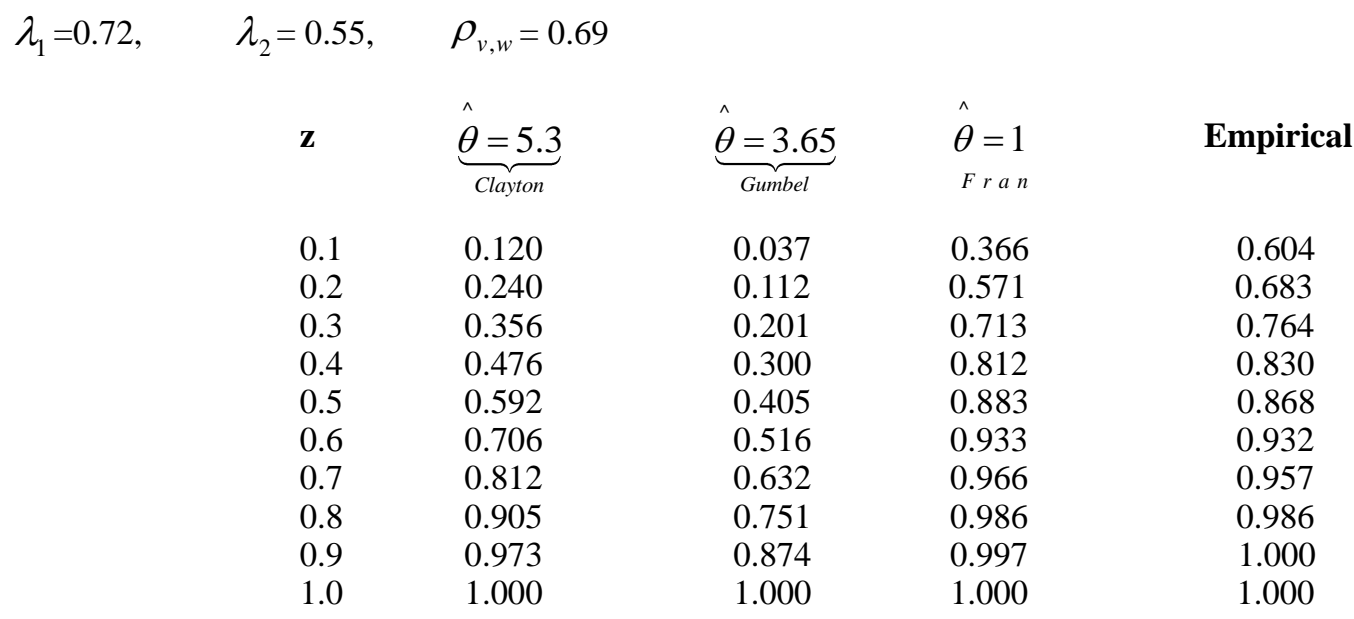

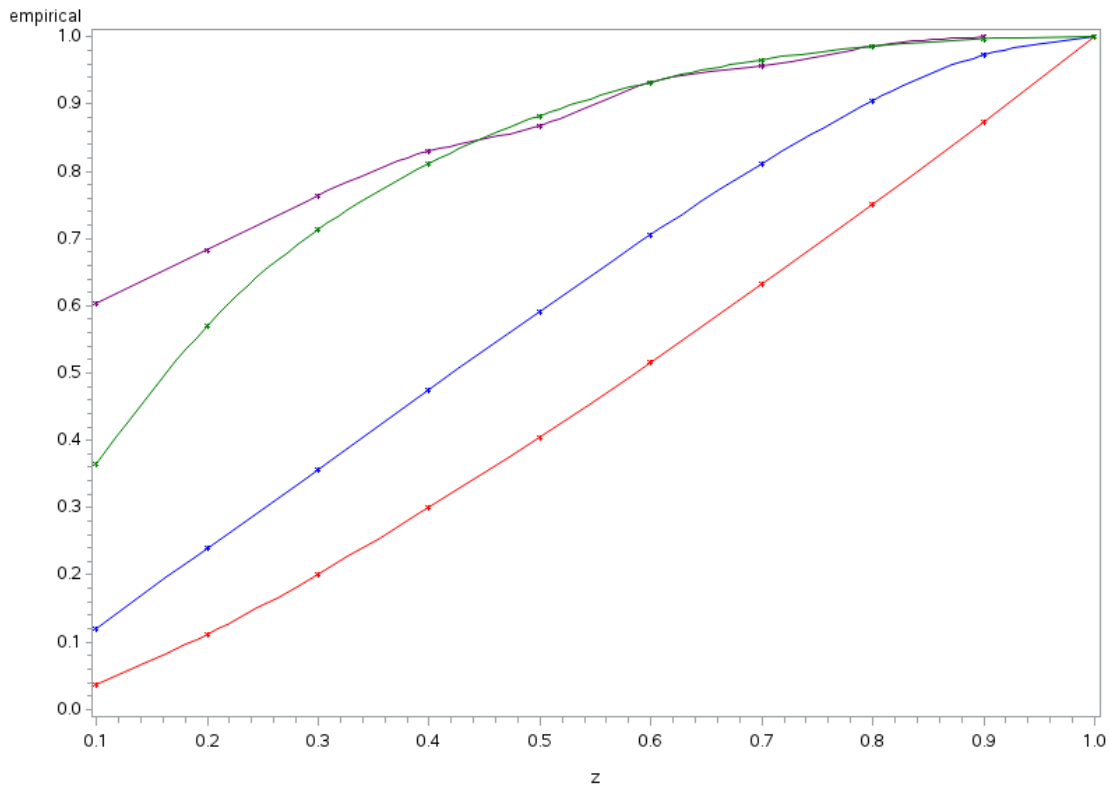


Simulation \#3

$\lambda_{1}=0.72, \quad \lambda_{2}=0.55, \quad \rho_{v, w}=0.96$

$\begin{array}{ccccc}\mathrm{z} & \underbrace{\hat{\theta}=49.282}_{\text {Clayton }} \underbrace{\hat{\theta=25.641}}_{\text {Gumbel }} \underbrace{\hat{\theta=0.4}}_{\text {Frank }} & \text { Empirical } \\ 0.1 & 0.102 & 0.091 & 0.344 & 0.417 \\ 0.2 & 0.204 & 0.187 & 0.541 & 0.558 \\ 0.3 & 0.306 & 0.286 & 0.682 & 0.782 \\ 0.4 & 0.408 & 0.386 & 0.785 & 0.803 \\ 0.5 & 0.510 & 0.486 & 0.861 & 0.828 \\ 0.6 & 0.612 & 0.588 & 0.917 & 0.873 \\ 0.7 & 0.714 & 0.690 & 0.956 & 0.914 \\ 0.8 & 0.816 & 0.793 & 0.982 & 0.939 \\ 0.9 & 0.918 & 0.896 & 0.996 & 0.966 \\ 1.0 & 1.000 & 1.000 & 1.000 & 1.000\end{array}$

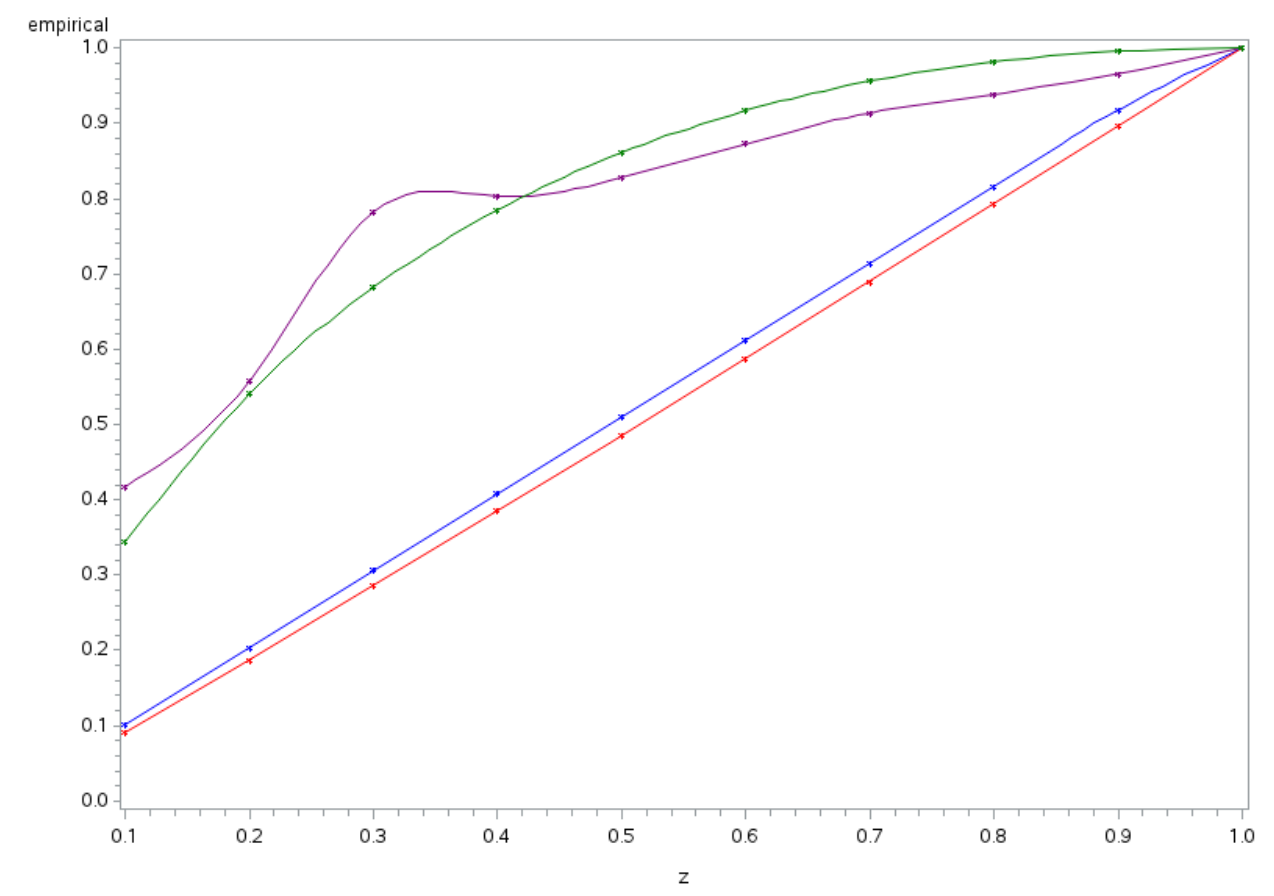


Simulation \#4

$\lambda_{1}=-0.72, \quad \lambda_{2}=0.55, \quad \rho_{v, w}=0.05$

$\begin{array}{lllll}\mathbf{z} & \underbrace{\hat{\theta}=-0.15}_{\text {Clayton }} \quad \underbrace{\hat{\theta}=0.925}_{\text {Gumbel }} & \underbrace{\hat{\theta=0.3}}_{\text {Frank }} & \text { Empirical } \\ 0.1 & 0.375 & - & 0.341 & 0.492 \\ 0.2 & 0.564 & - & 0.536 & 0.590 \\ 0.3 & 0.696 & - & 0.676 & 0.662 \\ 0.4 & 0.793 & 0.004 & 0.780 & 0.714 \\ 0.5 & 0.865 & 0.125 & 0.858 & 0.762 \\ 0.6 & 0.918 & 0.269 & 0.915 & 0.823 \\ 0.7 & 0.956 & 0.430 & 0.955 & 0.862 \\ 0.8 & 0.982 & 0.607 & 0.981 & 0.905 \\ 0.9 & 0.995 & 0.797 & 0.995 & 0.968 \\ 1.0 & 1.000 & 1.000 & 1.000 & 1.000\end{array}$

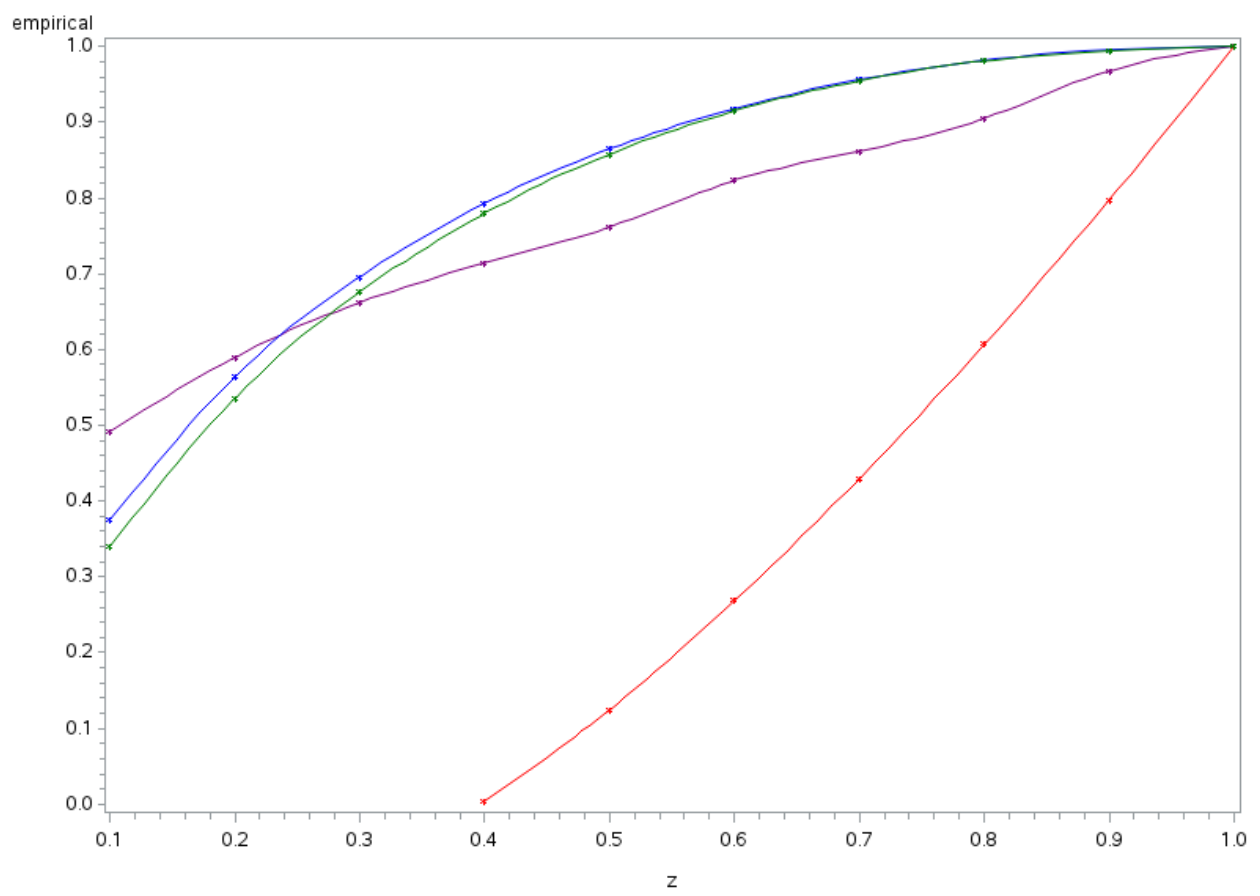


Simulation \#5

$\lambda_{1}=-0.72, \quad \lambda_{2}=0.55, \quad \rho_{v, w}=0.69$

$\begin{array}{llccr}\mathbf{z} & \underbrace{\hat{\theta}=1.817}_{\text {Clayton }} \quad \underbrace{\hat{\theta}=1.908}_{\text {Gumbel }} & \underbrace{\hat{\theta=0.26}}_{\text {Frank }} & \text { Empirical } \\ 0.1 & 0.154 & - & 0.339 & 0.519 \\ 0.2 & 0.304 & 0.031 & 0.534 & 0.626 \\ 0.3 & 0.447 & 0.111 & 0.674 & 0.694 \\ 0.4 & 0.578 & 0.208 & 0.778 & 0.760 \\ 0.5 & 0.697 & 0.318 & 0.856 & 0.810 \\ 0.6 & 0.801 & 0.439 & 0.914 & 0.853 \\ 0.7 & 0.884 & 0.569 & 0.954 & 0.898 \\ 0.8 & 0.947 & 0.706 & 0.981 & 0.941 \\ 0.9 & 0.986 & 0.850 & 0.995 & 0.986 \\ 1.0 & 1.000 & 1.000 & 1.000 & 1.000\end{array}$

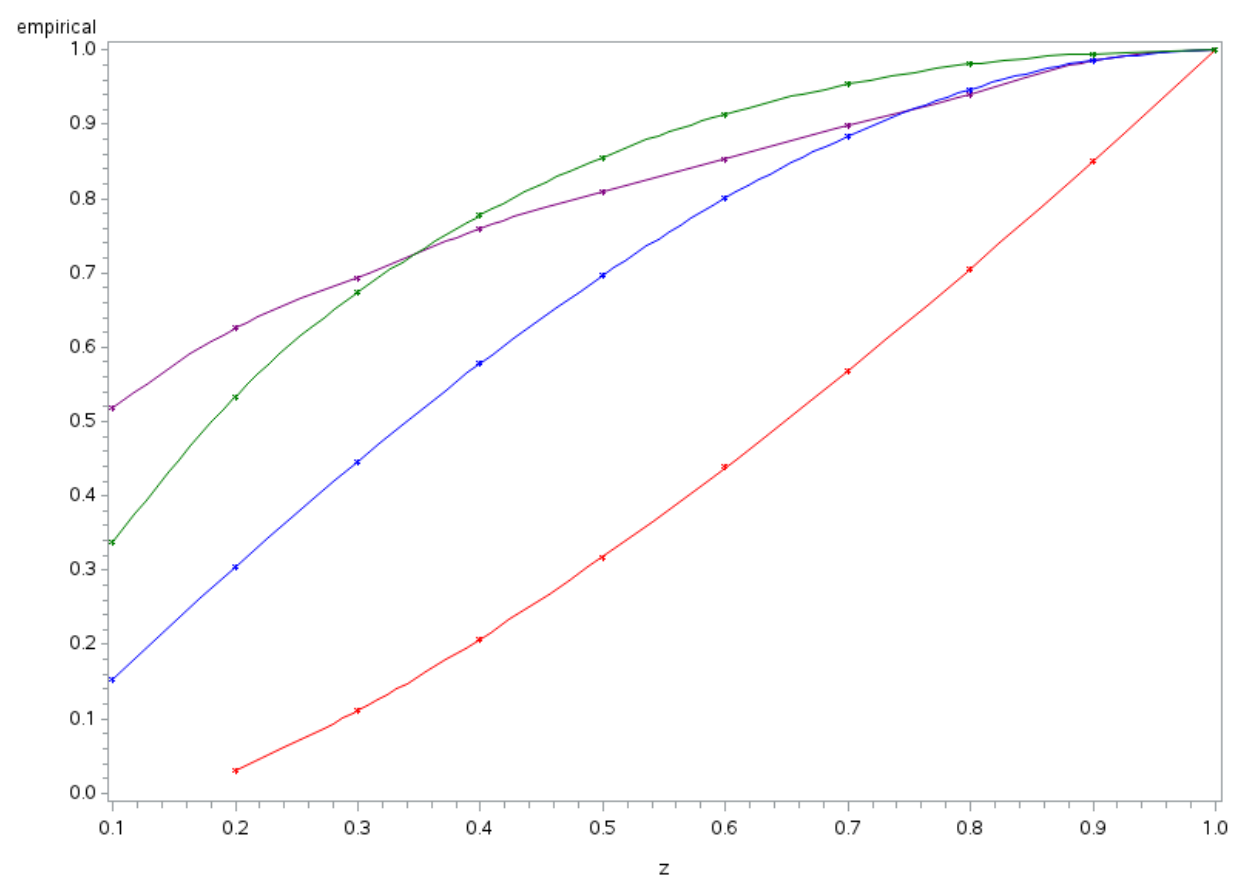


Simulation \#6

\begin{tabular}{|c|c|c|c|c|c|}
\hline \multirow[t]{13}{*}{$\lambda_{1}=-0.72$} & $\lambda_{2}=0.55$ & $\rho_{v, w}=$ & & & \\
\hline & $\mathbf{z}$ & $\hat{\theta}=4.92$ & $\hat{\theta}=3.46$ & $\hat{\theta}=0.25$ & Empirical \\
\hline & & $\overbrace{\text { Clayton }}$ & $\overbrace{\text { Gumbel }}$ & Frank & \\
\hline & 0.1 & 0.120 & 0.033 & 0.339 & 0.356 \\
\hline & 0.2 & 0.241 & 0.107 & 0.534 & 0.553 \\
\hline & 0.3 & 0.361 & 0.196 & 0.674 & 0.669 \\
\hline & 0.4 & 0.480 & 0.294 & 0.778 & 0.726 \\
\hline & 0.5 & 0.598 & 0.400 & 0.856 & 0.791 \\
\hline & 0.6 & 0.712 & 0.511 & 0.913 & 0.837 \\
\hline & 0.7 & 0.818 & 0.628 & 0.954 & 0.880 \\
\hline & 0.8 & 0.908 & 0.748 & 0.981 & 0.934 \\
\hline & 0.9 & 0.974 & 0.873 & 0.995 & 0.979 \\
\hline & 1.0 & 1.000 & 1.000 & 1.000 & 1.000 \\
\hline
\end{tabular}

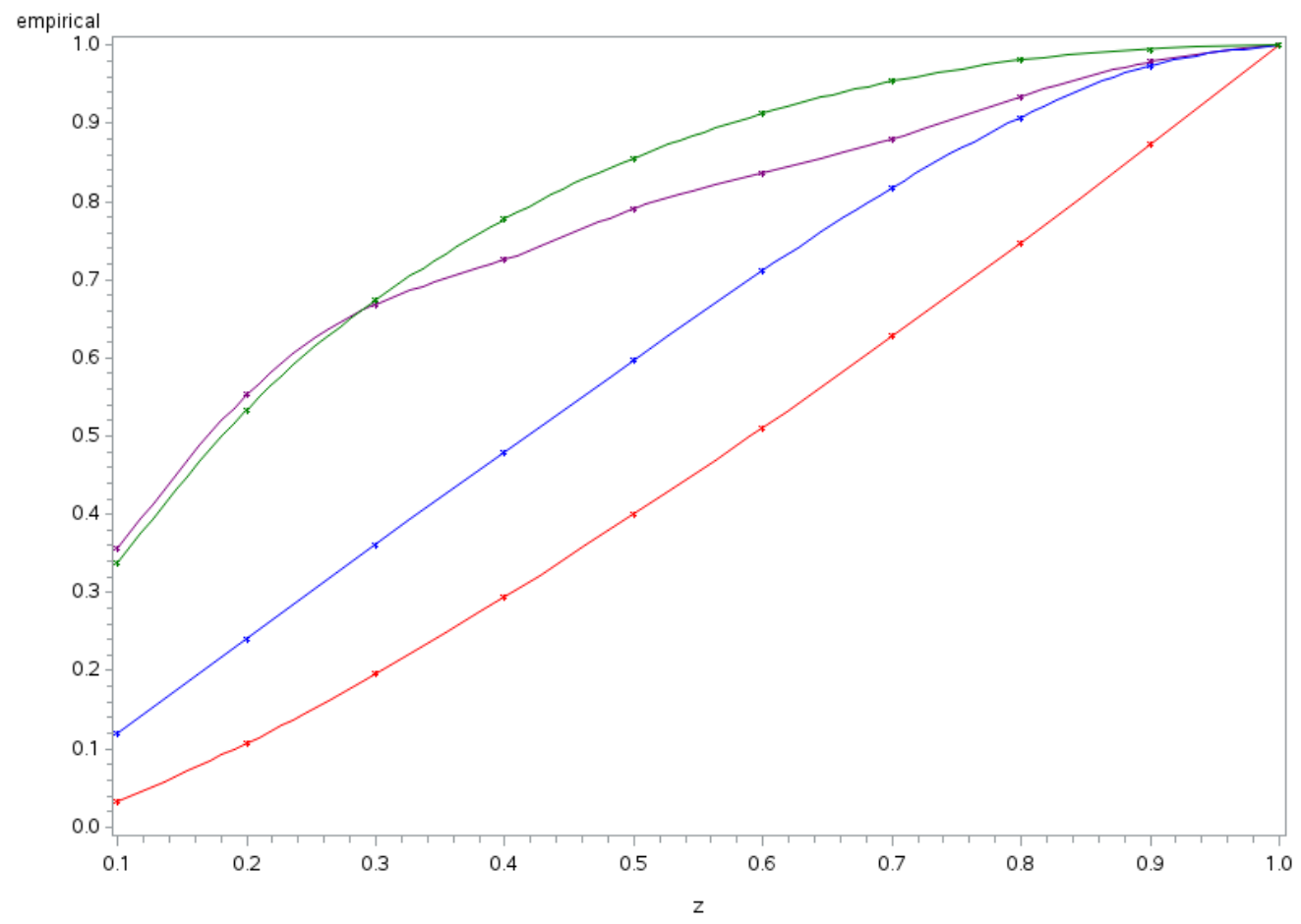


Simulation \#7

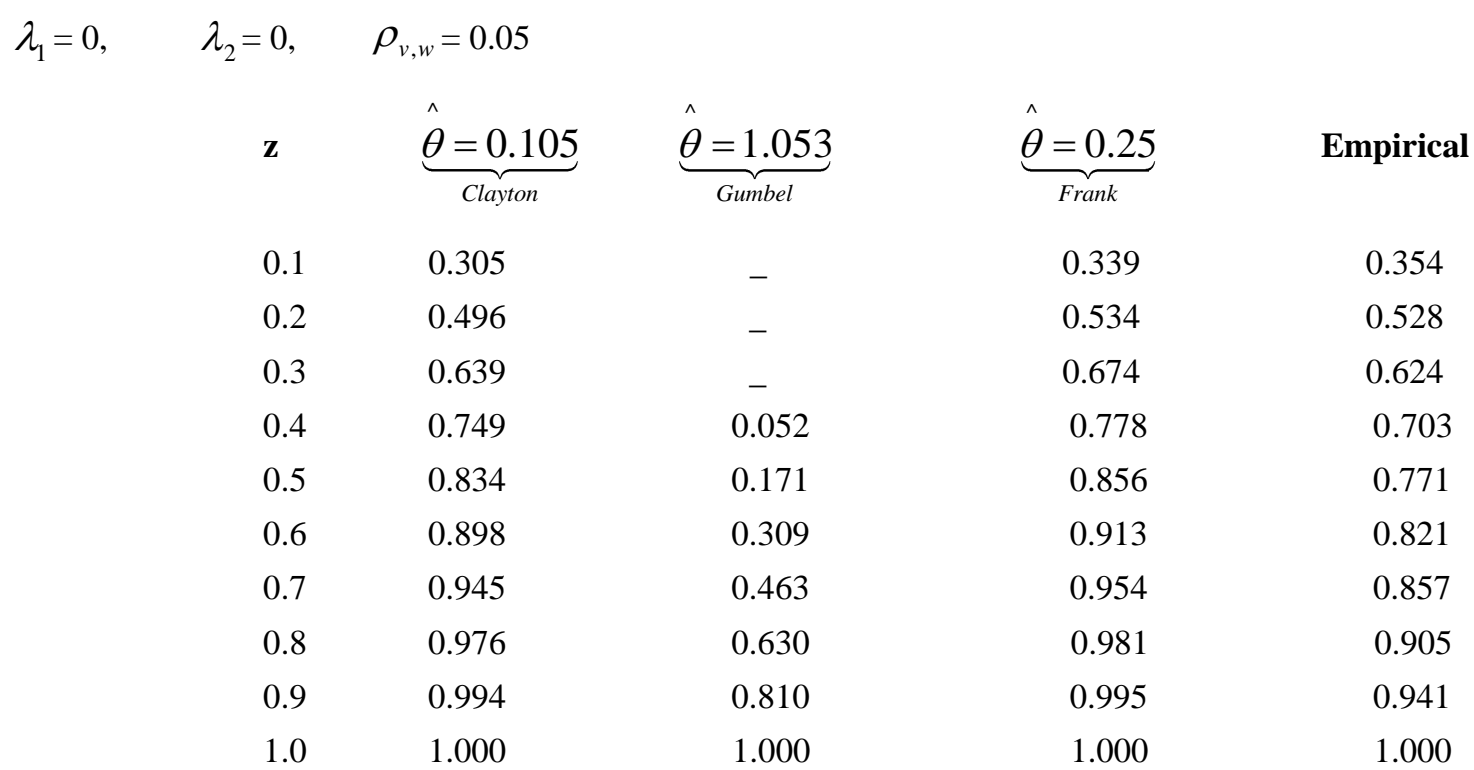

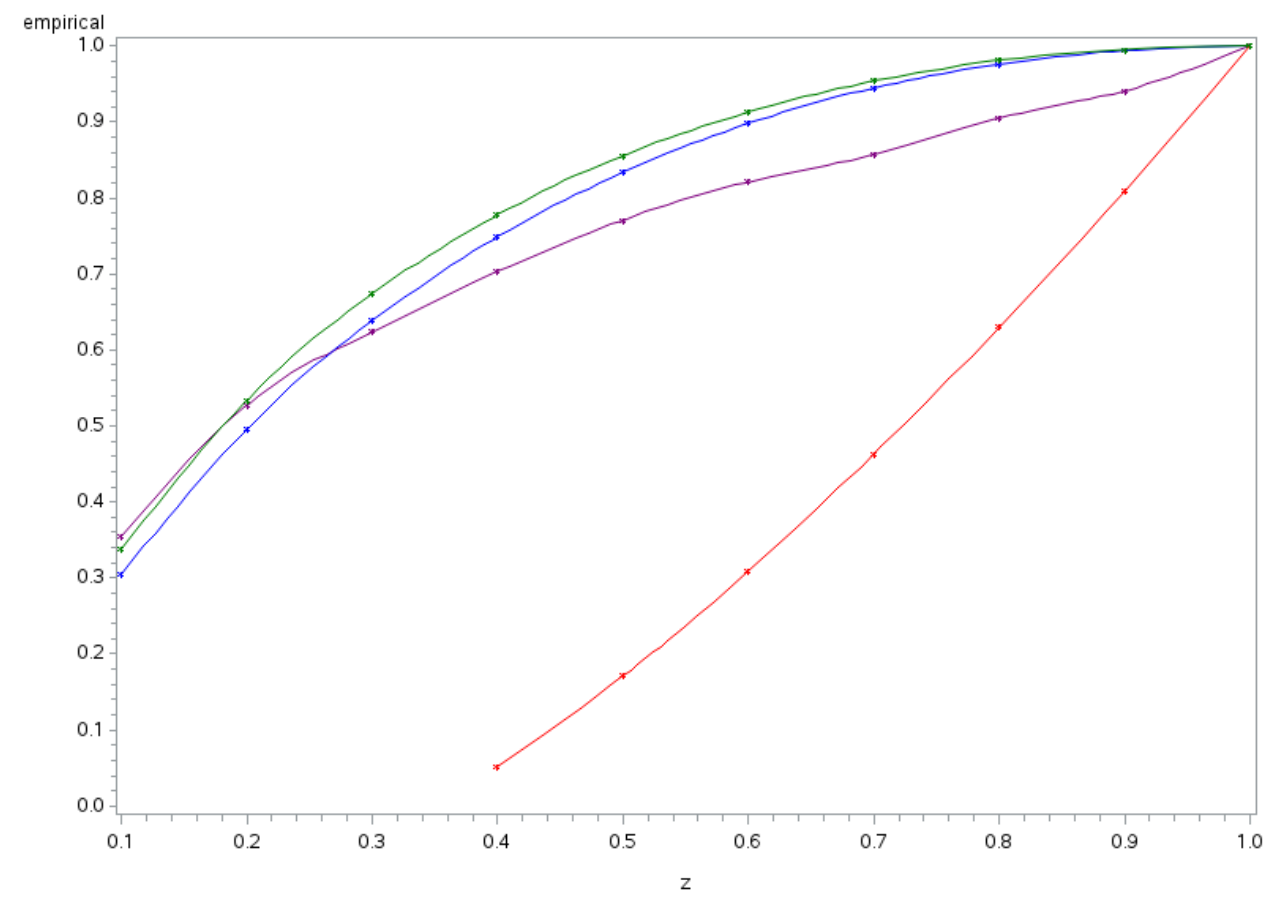


Simulation \#8

\begin{tabular}{|c|c|c|c|c|c|}
\hline \multirow[t]{12}{*}{$\lambda_{1}=0$} & $\lambda_{2}=0$ & $\begin{array}{l}\rho_{v, w}=0.69 \\
\hat{\underbrace{\theta}=4.452}\end{array}$ & $\underbrace{\hat{\theta}=3.226}$ & $\underbrace{\hat{\theta}=0.25}$ & Empirical \\
\hline & & $\overbrace{\text { Clayton }}$ & Gumbel & Frank & \\
\hline & 0.1 & 0.122 & 0.029 & 0.339 & 0.397 \\
\hline & 0.2 & 0.249 & 0.100 & 0.534 & 0.567 \\
\hline & 0.3 & 0.367 & 0.188 & 0.674 & 0.642 \\
\hline & 0.4 & 0.488 & 0.286 & 0.778 & 0.729 \\
\hline & 0.5 & 0.607 & 0.393 & 0.856 & 0.814 \\
\hline & 0.6 & 0.721 & 0.505 & 0.913 & 0.873 \\
\hline & 0.7 & 0.825 & 0.623 & 0.954 & 0.898 \\
\hline & 0.8 & 0.913 & 0.745 & 0.981 & 0.912 \\
\hline & 0.9 & 0.976 & 0.871 & 0.995 & 0.939 \\
\hline & 1.0 & 1.000 & 1.000 & 1.000 & 1.000 \\
\hline
\end{tabular}

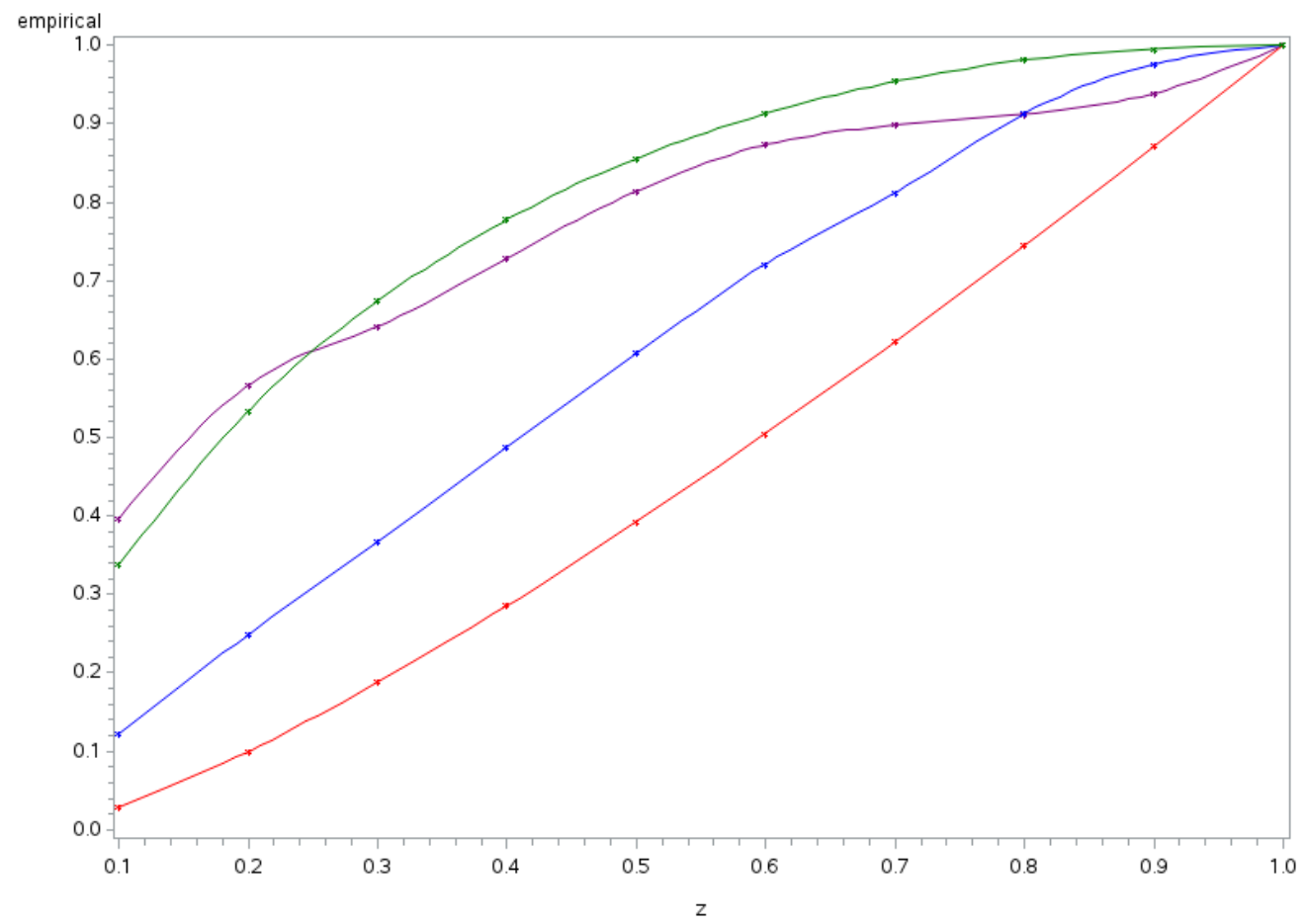


Simulation \#9

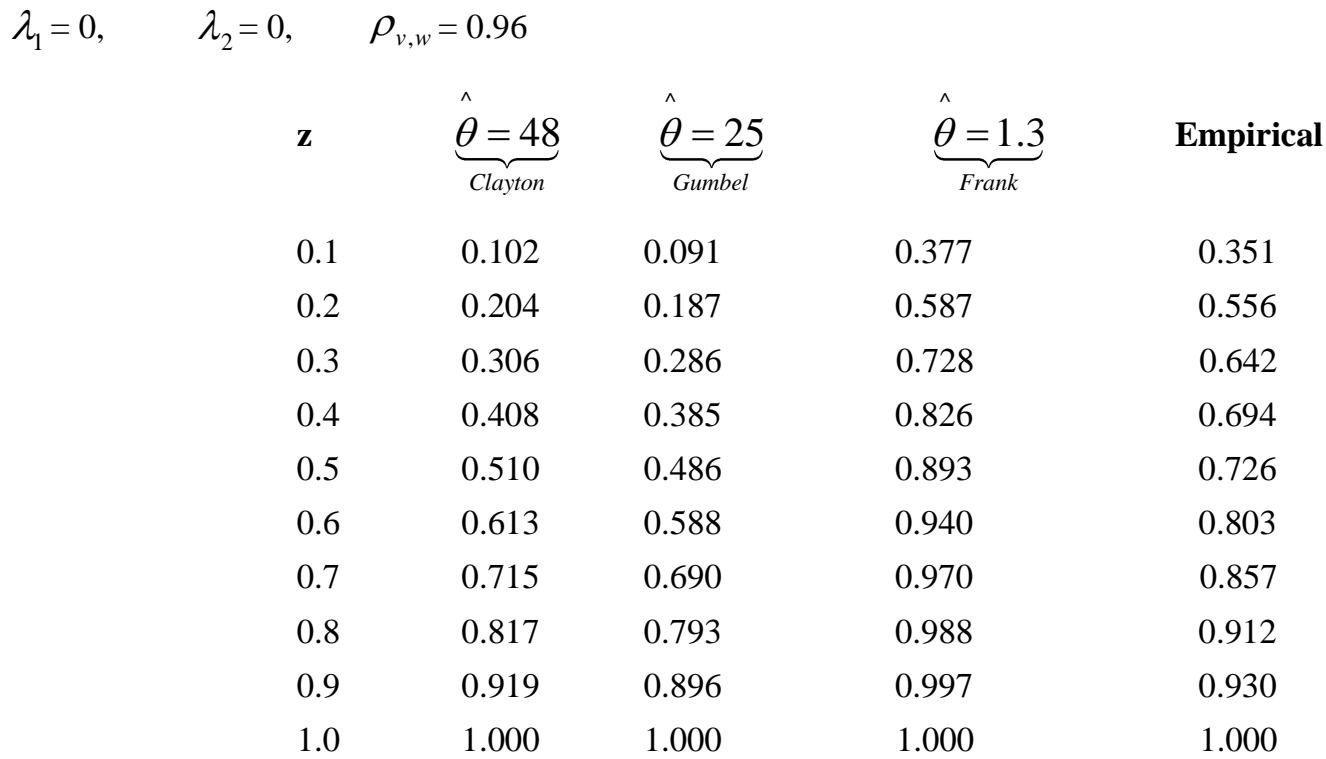

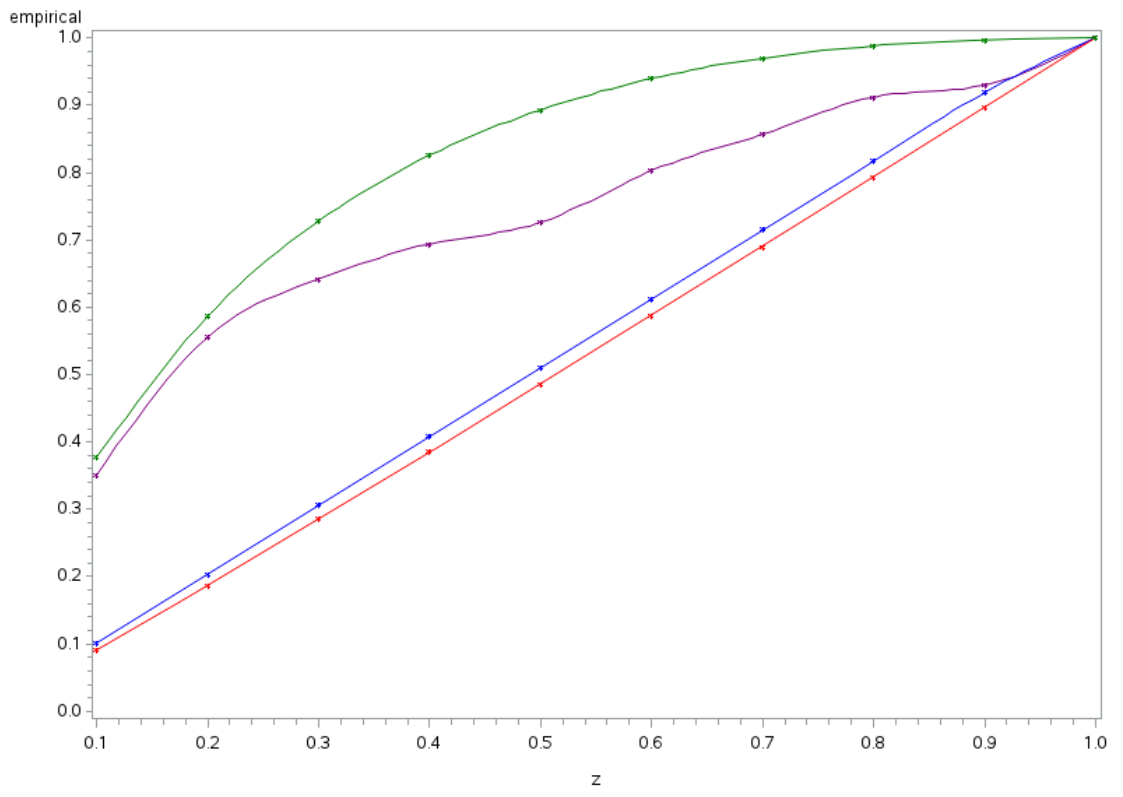

\section{Conclusion}

It is clear from our numerical study that the Frank Copula is a better Archimedean Copula for approximating a bivariate skew-normal distribution when the marginal distributions are skew-normal themselves. Also, it is clear from the study that Frank Copula is a better Archimedean Copula for approximating even a bivariate-normal distribution. It is easier to deal with the Frank Copula based density function compared to the density function of the bivariate skew-normal distribution as it involves lesser number of parameters. Due to this reason that an Archimedean Copula such as Frank Copula gives us an advantage when analyzing financial and the other types of data. Also, we can easily calculate the probabilities, ruin probabilities, conditional probabilities, quantiles and the conditional quantiles in the context of financial and other types of data.

\section{References}

Aziz, M. A. S. (2001). A Study of Unified Multivariate Skew Normal Distribution with Applications in Finance and Actuarial Science. Unpublished PhD dissertation, Bowling Green State University.

Azzalini, A. (1985). A Class of Distributions which includes the normal ones. Scandinavian Journal of Statistics, 12, 
171-178.

Azzalini, A. (1986). Further results on a class of distributions which includes the normal one. Statistica, 46, $199-208$.

Azzalini, A., \& Dalla, V. A. (1996). The multivariate skew-normal distribution. Biometrika, 83(4), 715-726. https://doi.org/10.1093/biomet/83.4.715

Brown, N. D. (2001). Reliability studies of the skew normal distributions. Electronic Theses and Dissertations, 408.

Charemza, W., Diaz, C., \& Makarova, S. (2015). Choosing the Right Skew Normal Distribution: the Macroeconomist' Dillema, No 15/08, Discsussion Papers in Economics, Department of Economics, University of Leicester.

Genest, C., \& MacKay, R. J. (1986). Copules Archimediennes et Familles de Lois Bidimensionnelles Don't les Marges Sont Donnes. The Canadian Journal of Statistics, 14, 145-159.

Gupta, A. K., Gonzalez-Farias, G., \& Dominguez-Molina, J. A. (2004). A multivariate skew normal distribution (Master's thesis, Department of Mathematics and Statistics, Bowling Green State University, Bowling Green, OH) (2004). Journal of Multivariate Analysis, 89(1), 181-190. https://doi.org/10.1016/S0047-259X(03)00131-3

Henze, N. (1986). A probabilistic representation of the 'skew-normal' distributionss. Scandinavian Journal of Statistics, $13,271-275$.

Krazanowski, W. J. (1988). Principles of Multivariate Analysis: A User's Perspective Oxford Statistical Series.

Nelsen, R. B. (2007). An Introduction to Copulas. Springer Series in Statistics.

Quiroga, A. M. (1992). Studies of the Polychoric Correlation and other Correlation Measures for Ordinal Variables. (PhD Thesis). Uppsala University.

Sklar, A. (1959). Fonctions de repartition a $n$ dimensions et leurs marges. Publications of the Institute of Statistics, University of Paris.

\section{Copyrights}

Copyright for this article is retained by the author(s), with first publication rights granted to the journal.

This is an open-access article distributed under the terms and conditions of the Creative Commons Attribution license (http://creativecommons.org/licenses/by/4.0/). 